\title{
Individual differences in the neural basis of causal inferencing
}

\author{
Chantel S. Prat ${ }^{\mathrm{a}, *}$, Robert A. Mason ${ }^{\mathrm{b}}$, Marcel Adam Just ${ }^{\mathrm{b}}$ \\ ${ }^{a}$ Department of Psychology and Institute for Learning E' Brain Sciences, University of Washington, P.O. Box 357988, Seattle, WA 98195-7988, United States \\ ${ }^{\mathrm{b}}$ Carnegie Mellon University, United States
}

\section{A R T I C L E I N F O}

\section{Article history:}

Accepted 30 August 2010

Available online 3 November 2010

\section{Keywords:}

Individual differences

Language

Inference

Right hemisphere

fMRI

Neuroscience

Discourse

Vocabulary

Reading skill

\begin{abstract}
A B S T R A C T
This study used fMRI to examine individual differences in the neural basis of causal inferencing. Participants with varying language skill levels, as indexed by scores on the vocabulary portion of the NelsonDenny Reading Test, read four types of two-sentence passages in which causal relatedness (moderate and distant) and presence or absence of explicit clause connectives were orthogonally varied to manipulate coherence and cohesion during inference generation. Skilled readers showed better neural efficiency (less activation) during all context sentences and during all inference conditions. Increased activation in lessskilled readers was most extensively distributed in the right hemisphere (RH) homologues of left hemisphere (LH) language areas, especially in the most difficult passage types. Skilled readers also showed greater sensitivity to coherence (greater activation and synchronization in moderately related than distantly related passages) whereas less-skilled readers showed sensitivity to cohesion (greater activation and synchronization when clause connectives were present than when they were not). These finding support the hypothesis that skilled reading comprehension requires recruitment of the RH on an "as needed" basis. We describe the dynamic spillover hypothesis, a new theoretical framework that outlines the conditions under which RH language contributions are most likely evoked.
\end{abstract}

(C) 2010 Elsevier Inc. All rights reserved.

\section{Introduction}

Individual differences in higher-level reading skill (such as those observed among literate college readers) are manifest in large part by differences in inferential abilities (Daneman \& Carpenter, 1980; Daneman \& Merikle, 1996; Just \& Carpenter, 1992; Long, Oppy, \& Seely, 1994, 1997). Skilled readers, for example, are more likely to generate inferences that are not essential for comprehending texts but nonetheless provide enriched discourse comprehension (Just \& Carpenter, 1992; Long et al., 1994, 1997; St. George, Mannes, \& Hoffman, 1997). The ability to generate such inferences is not only necessary for enriched discourse comprehension, but is also fundamental for more general reasoning abilities. The goals of this experiment were twofold: to determine the biological basis of individual differences in inference-making abilities, and to use these findings to evaluate existing theories of the neural underpinnings of inferential processes, with an emphasis on the role of the right hemisphere. To do so, we used fMRI to examine the relations between neural activation, cortical synchronization, and individual reading skill during comprehension of passages designed to elicit causal inferences.

The neural underpinnings of inferential processes are modulated by a complex interaction between reader characteristics

\footnotetext{
* Corresponding author. Fax: +1 2062216472.

E-mail address: csprat@uw.edu (C.S. Prat).
}

and text characteristics. The extent to which text characteristics (e.g., coherence and cohesion) modulate brain activation during inferencing has been well explored in the literature; however, less attention has been paid to how these characteristics interact with individual differences in comprehension ability. The current study addressed this intersection of reader and text variables by investigating how individuals of varying comprehension abilities are differentially influenced by manipulations of coherence and cohesion in passages designed to elicit causal inferences.

\section{Individual differences in neural efficiency}

A number of recent findings suggest that individual differences in cognitive abilities are characterized, in part, by differences in neural efficiency, measured by the amount of brain activation underpinning a given task (e.g., Haier et al., 1988; Maxwell, Fenwick, Fenton, \& Dollimore, 1974; Prat \& Just, in press; Prat, Keller, \& Just, 2007; Reichle, Carpenter, \& Just, 2000). In general, among healthy participants who can perform a task, higher cognitive abilities are indexed by less (more focal distribution or lower intensity) brain activation (see Neubauer and Fink (2009) for a review). For example, in a sentence-picture verification task, participants with higher verbal-working-memory capacities had lower activation volumes in typical language regions (e.g., Broca's area) when engaging in verbal strategies. Similarly, individuals with higher visual-spatial skills, as indexed by performance on 
mental rotation tasks, had lower activation volumes in typical visual association regions (e.g., parietal cortex) when engaging in spatial strategies (Reichle, Carpenter, \& Just, 2000). The lower activation volume in more proficient individuals can be interpreted as the use of fewer neural resources to perform a given set of computations, and hence can be thought of as neural efficiency.

Much of the research on individual differences in comprehension ability has reported increased efficiency in better readers (Maxwell et al., 1974; Prat \& Just, in press; Prat et al., 2007). Constraints on inference processes may arise, therefore, because less-skilled readers are utilizing more resources for basic comprehension processes, leaving fewer resources available for optional or elaborative processes. While these studies have used different indices of comprehension ability, a recent investigation of individual differences in sentence comprehension found that vocabulary knowledge, a crystallized index of reading experience, was a better predictor of neural efficiency than was verbal working memory capacity, a more general index of verbal information processing abilities (Prat \& Just, in press). In the current experiment, the relation between neural efficiency and language skill is examined by correlating the amount of brain activation during comprehension of passages that invite causal inference generation with measures of individual vocabulary size.

\section{Individual differences in neural synchronization}

Individual differences in inferential abilities may also vary as a function of the degree of coordination between component cortical subsystems in the language network. To function optimally, the areas responsible for executing subcomponent processes during reading must collaborate to synthesize the information necessary for comprehension. Such collaboration can be measured in functional neuroimaging studies by computing the correlation of the activation time series in a given region across time with the activation time series of another region. The extent to which the activation levels of two regions rise and fall in tandem is taken as a reflection of the degree to which the two regions are functionally connected, widely referred to as functional connectivity (Friston, 1994).

The degree of functional connectivity (synchronization) between the activated regions in a cortical network has been shown to reflect the network's effectiveness at the behavioral level. Bèuchel, Coull, and Friston (1999) found that in an extended learning paradigm, the functional connectivity increased sharply at those points in time when the behavioral performance also increased, indicating that the coordination between the cortical components of a system is an important facet of its effectiveness. Connectivity between regions also changes as a function of task demands. During discourse comprehension, for example, connectivity between left inferior frontal (Broca's area) and posterior superior temporal (Wernicke's area) increases when readers make inferential predictions (Chow, Kaup, Raabe, \& Greenlee, 2008).

The key linkage between functional connectivity and individual differences was provided by an investigation of sentence comprehension (Prat et al., 2007). This experiment found an interaction between individual differences in working memory capacity and task demands (manipulated in terms of syntactic and lexical complexity), such that high-capacity readers showed higher baseline functional connectivity in the language network, and either increased or maintained functional connectivity levels with increasing demand, whereas low-capacity readers' performance and functional connectivity often decreased with increasing task demand. The implication of these findings is that the efficacy of the language network is not only determined by the degree of activation of various regions but also by the extent to which activities between centers are coordinated. This paper will explore the hypothesis that individual differences in inferential abilities may be underpinned by differences in synchronization between the various cortical centers involved in component comprehension processes by comparing functional connectivity during comprehension as a function of individual reading experience.

\section{Neuroimaging investigations of inferential processes}

Inferential processes are central for establishing coherent discourse representations and thus have been the focus of numerous neuroimaging investigations of discourse comprehension. The brain regions reported, however, vary across investigations. This is not surprising due in part to the differences in types of inferences drawn (e.g., predictive versus bridging, required versus elaborative), conditions supporting inferential processes (e.g., cohesion and coherence), type of texts (e.g., short, two-sentence passages versus longer, multi-sentence passages), and methods used (e.g., listening versus reading, passive comprehension versus explicit relatedness judgments). Despite the heterogeneous nature of inferential processes and methods for assessing them, both commonalities and inconsistencies emerge from the research.

The left hemisphere ( $\mathrm{LH}$ ) perisylvian language regions (including Broca's and Wernicke's areas) and medial prefrontal cortex (mPFC) have been repeatedly implicated in studies of inferencing (Chow et al., 2008; Ferstl \& von Cramon, 2001; Friese, Rutschmann, \& Raabe, 2008; Kuperberg, Lakshmanan, Caplan, \& Holcomb, 2006; Sieborger, Ferstl, \& von Cramon, 2007). Increased LH activation for inferred or implied over explicitly stated events has been reported in the literature (Kuperberg et al., 2006; Virtue, Haberman, Clancy, Parrish, \& Jung Beeman, 2006). Similarly the role of mPFC has been consistently established. The consensus on the role of $\mathrm{MPFC}$ in inferencing is that it plays a general role in coherence monitoring during comprehension (for reviews see Ferstl, Neumann, Bogler, \& von Cramon, 2008; Mason \& Just, 2006) and that during inferential processes this becomes important because it signals a coherence break (Chow et al., 2008; Ferstl \& von Cramon, 2001; Mason \& Just, 2006; Sieborger et al., 2007) that initiates the inference generation process.

In contrast, the role of the right hemisphere $(\mathrm{RH})$ in inferential processes remains controversial. Although it is generally accepted that the RH contributes to discourse-level reading comprehension, the nature and timing of its contribution remains uncertain. Some neuropsychological research on $\mathrm{RH}$-damaged patients suggests that the RH is involved in various types of inferential processes (Beeman, 1993a; Brownell, Potter, Bihrle, \& Gardner, 1986). Other investigations, however, fail to find deficits in $\mathrm{RH}$-damaged patients, even when attempting to replicate previous studies (McDonald \& Wales, 1986; Tompkins, 1991; Tompkins, Fassbinder, Lehman Blake, Baumgaertner, \& Jayaram, 2004). Some neuroimaging studies of healthy controls also report $\mathrm{RH}$ contributions to inferential processes, especially in the inferior frontal gyrus and in middle and superior temporal gyri (e.g., Kuperberg et al., 2006; Mason \& Just, 2004; Sieborger et al., 2007; Virtue, Parrish, \& Jung-Beeman, 2008; Virtue et al., 2006). In an fMRI investigation of the influence of coherence and cohesion on inferences, however, Ferstl and von Cramon (2001) found no differences in RH activation as a function of factors influencing inferential processes. In addition, a recent meta-analysis of discourse comprehension studies found no unique RH contributions to inferential processes (Ferstl et al., 2008).

Explanations about the implications of $\mathrm{RH}$ activation vary across studies. For example, Jung-Beeman and colleagues (e.g., Beeman, Bowden, \& Gernbacher, 2000; Jung-Beeman, 2005) propose that the $\mathrm{RH}$ processes all language in parallel with the dominant $\mathrm{LH}$, but that its unique processing style (coarse coding) gives it advantages over the LH when activation of diffuse semantic fields is advantageous (e.g., during unconstrained predictive 
inferences and metaphor comprehension). According to JungBeeman, both the $\mathrm{LH}$ and $\mathrm{RH}$ are involved in activation of semantic fields, selection of appropriate meanings, and integration of meanings into the discourse representation (Jung-Beeman, 2005). Mason and Just (2004), on the other hand, proposed that the RH becomes increasingly involved when successfully drawn inferences are integrated into text representations. They found that moderately related two-sentence passages (with sufficient constraints to allow successful inference selection and integration) resulted in higher $\mathrm{RH}$ activation than both highly related passages (where no inference was required) and distantly related passages (where lack of sufficient constraints may have prevented successful inference selection). Kuperberg et al. (2006) also showed increased RH activation, especially in the inferior frontal gyrus, for moderately related over both distantly and closely related sentence pairs, but found increased $\mathrm{RH}$ activation in right temporal regions for distantly related sentence pairs over moderately related pairs. One alternate hypothesis explored in this experiment is that $\mathrm{RH}$ activation observed during inferential processes is merely an index of the cognitive difficulty experienced during reading (e.g., Reichle \& Mason, 2007).

\section{The dynamic RH spillover hypothesis}

The somewhat inconsistent findings from these and other attempts to characterize RH language function suggest that the conventional theoretical framework may lack a consideration of an adaptive, "as needed" role of the RH in language processes. We propose the dynamic RH spillover hypothesis, which describes the conditions that predict $\mathrm{RH}$ participation in language comprehension processes. Briefly stated, the dynamic RH spillover hypothesis proposes that with respect to linguistic processes: (1) the $\mathrm{RH}$ serves as a resource reserve for language processing with similar but coarser-grained and less efficient capabilities than the dominant LH homologues and (2) the RH becomes increasingly engaged when the processing demands of a language task outstrip the resources available in LH such that some of the residual processing spills over into RH (Just, Carpenter, Keller, Eddy, \& Thulborn, 1996; Just \& Varma, 2007).

The idea that lateralized processes "spill over" into contralateral hemispheres with increased difficulty is not a new one. Just and colleagues (1996) for example, found increased RH activation during sentence comprehension when syntactic complexity increased. Such spillover phenomena are not limited to the $\mathrm{RH}$; a recent experiment by Mitchell and Ross (2008) showed that prosodic processes (normally lateralized to the $\mathrm{RH}$ ) drew increasingly upon $\mathrm{LH}$ homologues as complexity increased.

Individual differences research is central to this theory because it predicts that recruitment of the RH varies systematically across people, depending on an individual's capabilities and on the amount of demand imposed by the task relative to the available resources. According to this view, the LH language areas are fairly consistently evoked in most people for a given language task, whereas RH contributions are more variable between participants and tasks and are therefore more difficult to characterize at the group level. This difficulty should be surmountable, however, with the application of a theoretical framework that provides a systematic account of the individual differences in $\mathrm{RH}$ involvement in language processing.

Variability in right hemisphere contributions as a function of comprehension ability has been observed in a variety of language comprehension paradigms. For example, in a series of dividedvisual-field experiments investigating discourse representation, Prat, Long, and Baynes (2007) found that less-skilled readers showed evidence of sentence-level (proposition) and discourselevel (topic) representations in both the right and left hemispheres, whereas skilled readers showed only LH priming of these representations. In an fMRI experiment, Mason and Just (2007) found increased $\mathrm{RH}$ activation in low-working-memory-capacity participants during lexical ambiguity resolution. Thus, it is plausible that the degree to which the $\mathrm{RH}$ is involved in inference generation varies as a function of factors influencing individual comprehension ability.

In the current experiment, we investigate the neural underpinnings of inferential processes in readers with varying experience levels, under conditions that have been shown to modulate the ease with which causal inferences can be generated (coherence and cohesion). According to the spillover hypothesis, increased RH activation should be observed in conditions where comprehension of passages is most difficult. One straightforward prediction of this hypothesis is that less-experienced (low vocabulary) readers should have greater $\mathrm{RH}$ activation during inference generation and potentially during baseline sentence comprehension processes.

With respect to coherence, behavioral research suggests that less-coherent (unrelated) passages take longer to read and are harder to remember than more coherent (moderately related) passages (Keenan, Baillet, \& Brown, 1984; Myers, Shinjo, \& Duffy, 1987) and thus may draw more heavily upon RH resources; however, in the absence of explicit instructions to do so, less-skilled readers may not attempt to generate inferences linking distantly related sentences (optional inferences). One way to trigger inferential processes between two distantly related sentences is to include clause connectives such as "because" that explicitly indicate a causal relation between the two sentences (e.g., Millis \& Just, 1994). Ferstl and von Cramon (2001) found behavioral and brain activation evidence that the presence of connectives (cohesion) facilitated processing of coherent passages but made processing of incoherent passages more difficult. Thus, according to the spillover hypothesis, we should see the most $\mathrm{RH}$ activation in distantly related passages with connectives, and the least RH activation in the moderately related passages with connectives.

One goal of these manipulations is to understand which factors are most directly related to the role of the $\mathrm{RH}$ in inference generation, and at what level of information processing the $\mathrm{RH}$ becomes involved. A broader goal of the current experiment is to determine the biological basis of constraints on inference generation abilities.

\section{Method}

\subsection{Participants}

Data were analyzed from 18 right-handed, native-English speaking paid undergraduate volunteers recruited through Carnegie Mellon University, ranging in age from 19 to 25 years. Twenty participants were run ( 12 female) with one participant excluded from analyses due to excessive head motion $(>2 \mathrm{~mm}$ ) and another participant excluded for poor (chance-level) performance on comprehension items. Nelson-Denny Vocabulary Test Scores ranged from 54 th to 97 th percentile (mean $=83$, sd $=11.09$ ). Scores of males and females did not differ $[t(16)=.53, p>.50]$. All participants gave informed consent before participating.

\subsection{Materials}

The materials consisted of 46 two-sentence passages. Forty of these were experimental passages, and six were filler items that were followed by comprehension questions. The experimental passages were pairs of sentences that varied in their degree of causal relatedness (moderate or distant), taken from Mason and Just (2004), who used a subset of the materials described in Myers et al. (1987). These passages have been studied previously and 
Table 1

Sample stimuli.

Moderately related sentences

Sandra walked barefoot on the littered beach

(Consequently) she had to clean out the wound on her foot

Distantly related sentences

Tom decided to run a marathon for charity.

(Consequently) he had many visitors at the hospital.

normed behaviorally, and were only modified by the addition of a clause connective to the beginning of the second sentence (such as "Consequently...") of half the passages. This resulted in four conditions in which the two degrees of causal relatedness between sentences and the presence or absence of a clause connective were orthogonally varied, with ten passages of each of the four conditions occurring in the stimulus list (see Table 1 for sample passages). The moderate (coherent) and distantly related (lesscoherent) passages were balanced for word length (mean number of words $=18$ and 17.5 respectively, sem $=.39$ and .38 respectively) and due to the addition of a connective, the cohesive passages were an average of one word longer than the incohesive passages (mean number of words $=17.95$ and 16.6 respectively, sem $=.30$ and .23 , respectively). The order of the passages was the same for all participants, with the experimental conditions presented in pseudorandom order such that no two passages of the same type occurred back to back and filler passages occurred at approximately even intervals throughout the experiment.

\subsection{Procedure}

During the fMRI scan, participants were instructed to read each passage for comprehension and to answer the Yes-No comprehension questions as they appeared. Comprehension questions were analyzed to insure that the subjects were attending to the stimuli. The first sentence of each experimental passage was presented for $5 \mathrm{~s}$, and then it disappeared. The second sentence appeared for $5 \mathrm{~s}$, and then it disappeared and was followed by a blank rest period of $9 \mathrm{~s}$, as shown in Fig. 1.

For filler passages, a comprehension question followed the second sentence, appearing for $5 \mathrm{~s}$. Participants pressed a mouse button corresponding to either yes or no in response to the comprehension question, which tested awareness of information explicitly mentioned in the passage. A rest period of $9 \mathrm{~s}$ followed each comprehension question. Four 30-s baseline periods, during which participants were instructed to relax and clear their minds while viewing an $X$ on the screen, occurred at the beginning, end, and evenly spaced throughout the experiment. The sentence displays, subtending a visual angle of approximately $30^{\circ}$, were projected onto a screen attached to the bore of the scanner behind the participant's head and were viewed through mirrors attached to the head coil.

The participants underwent behavioral testing and practice one or two days prior to their scan. The behavioral tests consisted of the 80-item vocabulary portion of the Nelson-Denny Reading Test (Riverside Publishing Company), the Reading Span Test (Daneman \& Carpenter, 1980), ${ }^{1}$ and the Edinburgh Handedness Inventory (Old-

\footnotetext{
${ }^{1}$ Daneman and Carpenter Reading Span Test scores are collected on all participants seen in this laboratory; however we did not analyze scores for the current study as we did not have a representative range of scores (1 low-span participant, 11 averagespan participants, and 7 high-span participants). Nelson-Denny Vocabulary scores and Reading Span scores were not correlated $(r=-.13, p<.10)$ in this sample, and have not been correlated in the larger group of over 200 participants run in our laboratory in the past five years. While the absence of a relation between these variables is somewhat inconsistent with the literature and may be idiosyncratic of the Carnegie Mellon population, it is not limited to this sample of participants. Please see Prat \& Just (in press) for a more thorough description of the differences in brain and behavior measured by these two indices of comprehension ability.
}

field, 1971). The practice consisted of performing the comprehension task in a mock scanner using a separate set of stimuli.

\section{4. fMRI data acquisition}

The data were collected using a Siemens Allegra 3.0 T scanner at the Brain Imaging Research Center jointly operated by Carnegie Mellon University and the University of Pittsburgh. The study was performed with a gradient echo planar pulse sequence with $\mathrm{TR}=1000 \mathrm{~ms}, \mathrm{TE}=30 \mathrm{~ms}$, and $\mathrm{a} 60^{\circ}$ flip angle. Seventeen obliqueaxial slices were imaged with an interleaved acquisition, and each slice was $5 \mathrm{~mm}$ thick with a gap of $1 \mathrm{~mm}$ between slices. The resulting acquisition matrix of $64 \times 64$ with $3.125 \times 3.125 \times 5 \mathrm{~mm}$ voxels covered the majority of the brain with the exception of the most anterior portion of the temporal lobe, the most anterior portion of the orbital frontal lobe, and the inferior portion of the cerebellum, with some variation across participants.

\subsection{Voxel-wise analyses}

The data were analyzed using SPM2 (Wellcome Department of Imaging Neuroscience, www.fil.ion.ucl.ac.uk/spm) to examine the distribution of activation during passage comprehension as a function of vocabulary size. Images were corrected for slice acquisition timing, motion-corrected, normalized to the Montreal Neurological Institute (MNI) template, resampled to $2 \times 2 \times 2 \mathrm{~mm}$ voxels, and smoothed with an $8 \mathrm{~mm}$ Gaussian kernel to decrease spatial noise. Statistical analyses were performed on individual and group data using the general linear model as implemented in SPM2 (Friston, Frith, Frackowiak, \& Turner, 1995).

Response to comprehension questions were analyzed first to insure that subjects were attending to all stimuli. Most participants got all filler questions correct (mean accuracy $=94.4 \%$, sem $=$ $2.7 \%$ ), but the one participant who responded around chance-level was removed from analysis. Data from all 46 passages was analyzed for the remaining participants. The four inference conditions were time locked to the presentation of the second sentence, the earliest point at which an individual could generate an inference. For individual participants, a fixed-effects model that incorporated a high-pass filter with a cut-off of 378 s and an AR(1) correction for serial autocorrelation was used to estimate parameters. Group analyses were performed using a random-effects model. Contrasts reflecting group differences in the distribution of activation were computed, calculating the four inference conditions relative to fixation across conditions, the coherence effect (collapsing across clause connective conditions), the cohesion effect (collapsing across causal relatedness conditions) and the interaction of coherence and cohesion (distant cohesive $>$ incohesive - moderate cohesive $>$ incohesive). The relationship between language skill and brain activation was assessed on a voxel-wise basis using a random-effects simple regression model in which Nelson-Denny Reading Test scores served as the independent variable and the above contrast values on individual participants served as the dependent variable. A height threshold of $p<.001$ uncorrected and an extent threshold of 12 voxels (corresponding to two voxels in original space) were used on all analyses.

\subsection{Volumetric analyses}

Twelve functionally defined regions of interest (ROIs) were selected from a larger group of 21 spherical ROIs previously defined to encompass all of the major regions of activation across five discourse comprehension experiments conducted in this laboratory. We chose ROIs that have been reported to be important in inferential processes including bilateral inferior frontal and temporal regions, a medial prefrontal region, and a region in the precuneus. 


\section{Schematic Depiction of the Presentation of an Inference Passage}

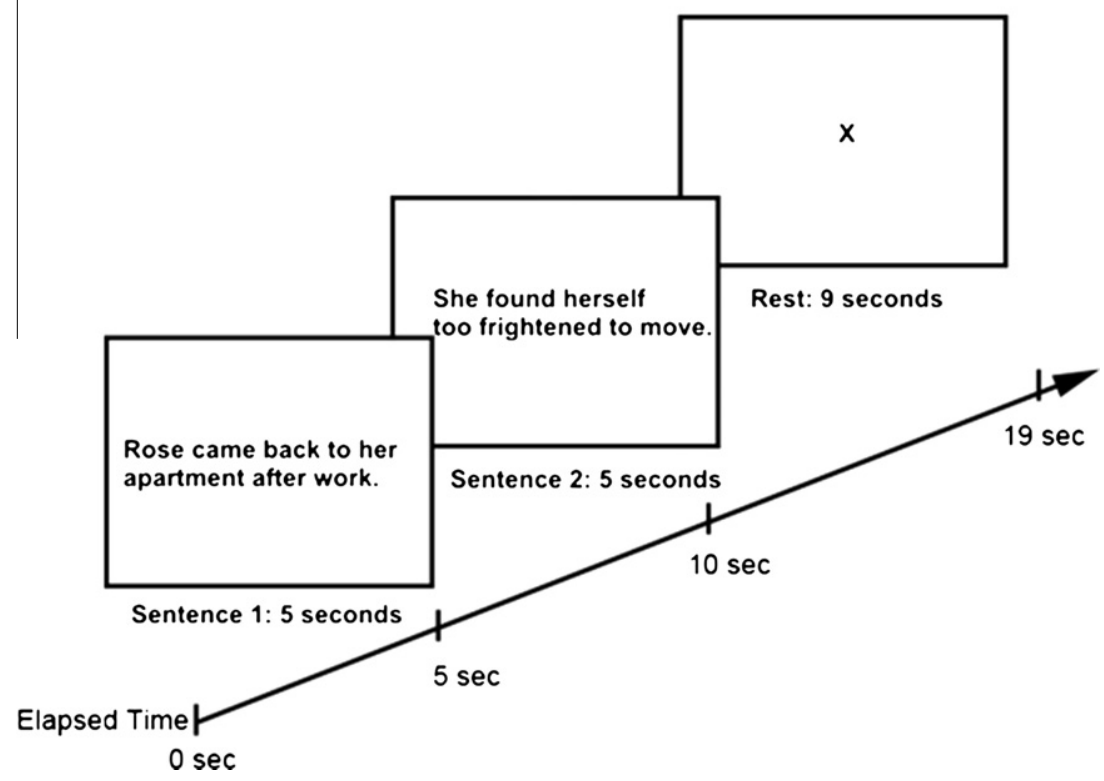

Fig. 1. Schematic depiction of the presentation of one of the inference passages.

Table 2

Locations of 12 regions of interest used in network connectivity analyses.

\begin{tabular}{|c|c|c|c|c|c|c|}
\hline \multirow[t]{2}{*}{ Function } & \multirow[t]{2}{*}{ Cortical region } & \multirow[t]{2}{*}{$\mathrm{BA}$} & \multirow[t]{2}{*}{ Radius } & \multicolumn{3}{|c|}{ Centroid MNI coordinates } \\
\hline & & & & $x$ & $y$ & $z$ \\
\hline \multirow[t]{3}{*}{ LH frontal/selection } & LH inferior frontal gyrus (inferior orbital) & 47 & 12 & -48 & 30 & -10 \\
\hline & LH inferior frontal gyrus (middle) & 45 & 14 & -48 & 18 & 18 \\
\hline & LH sup inferior/middle frontal gyri & 6 & 14 & -40 & 2 & 52 \\
\hline \multirow[t]{2}{*}{ LH temporal/semantic } & LH middle temporal & 41 & 14 & -56 & -22 & 12 \\
\hline & LH posterior superior temporal & 40 & 14 & -52 & -54 & 18 \\
\hline LH integration & LH anterior temporal & 38 & 14 & -50 & 10 & -26 \\
\hline \multirow[t]{2}{*}{ RH frontal/spillover/selection } & RH inferior frontal gyrus (inferior orbital) & 47 & 14 & 52 & 30 & -14 \\
\hline & RH inferior frontal gyrus (middle) & 46 & 14 & 48 & 22 & 26 \\
\hline \multirow[t]{2}{*}{ RH temporal/spillover/semantic } & RH middle temporal & 20 & 18 & 56 & -8 & -24 \\
\hline & RH posterior temporal & 22 & 18 & 48 & -50 & 6 \\
\hline Coherence monitoring & Medial prefrontal & 9 & 14 & -6 & 56 & 40 \\
\hline Memory & Precuneus & & 14 & -2 & -60 & 46 \\
\hline
\end{tabular}

Because this was an individual differences study and previous research suggests that RH activation varies (both in extent and location) between individuals, we modified the RH ROIs, decreasing the number and increasing the size of ROIs, to capture the most activation in individual participants. MNI coordinates, Brodmann's Areas, and sphere radii for each of the 12 ROIs are listed in Table 2.

The number of voxels activated (volume) in each ROI above the height threshold of $p<.001$, uncorrected, was calculated for each participant independently, for each of the four inference conditions. The data for each ROI were analyzed separately using 2 (coherence) $\times 2$ (cohesion) ANOVAs, with Nelson-Denny Reading Test scores serving as between participants covariates. Coherence and cohesion were within subjects variables. All effects were tested at a significance level of $p<.05$, unless otherwise indicated.

\subsection{Functional connectivity analysis}

The 12 functionally defined ROIs described above were into seven theoretical functional units: a LH temporal/semantic network (consisting of middle and posterior superior temporal ROIs), a LH frontal/selection network (consisting of the three lateral frontal
ROIs), a RH temporal/semantic (or spillover) network (consisting of middle and posterior superior temporal ROIs), a RH frontal/ selection (or spillover) network (consisting of the two lateral frontal ROIs), a coherence monitoring node (consisting of the medial frontal ROI), an integration node (consisting of the left anterior temporal ROI), and a memory node (consisting of the precuneus ROI). Time courses were extracted for each participant individually, averaged over only those voxels within each ROI that were activated above a threshold of $p=.001$, uncorrected, in any of the four passage conditions. The input data were the normalized and smoothed images that had been low-pass filtered and had the linear trend removed. Network-level connectivity analysis was conducted such that the average functional connectivity of each pair of ROIs within a functional network or between two functional networks was computed for each participant. Any participants with fewer than 12 activated voxels in either of the ROIs constituting a pair were excluded from that analysis. Fisher's $r$ to $z$ transformation was applied to the correlation coefficients for each participant prior to averaging and correlational analyses. The functional connectivity for each network pair was analyzed with the same ANOVA procedure used in the volumetric analyses. 


\section{Right Hemisphere Activation during Inferencing as a function of Coherence and Cohesion}

\author{
Cohesive: \\ Clause Connective
}

\author{
Less-Coherent: \\ Distantly Related \\ Coherent: \\ Moderately Related
}

\section{Less Cohesive:}

No Connective
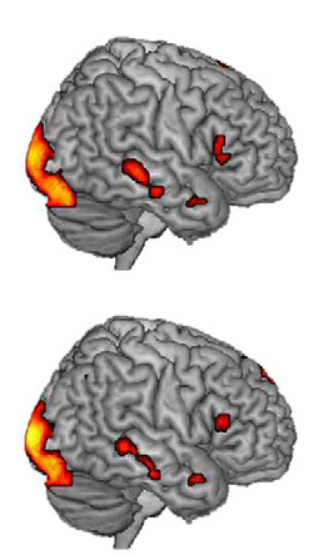
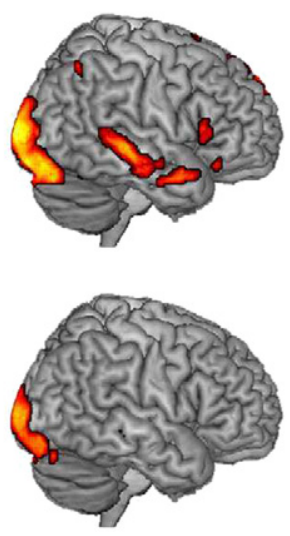

Fig. 2. Activation map showing distribution of activation in the right hemisphere for the reliable contrast of each of the four inference conditions with fixation.

\section{Results}

\subsection{Coherence and cohesion in the right hemisphere}

Distribution of activation analyses at the group level showed that all passage types resulted in some degree of $\mathrm{RH}$ activation. ${ }^{2}$ As predicted by the results of Ferstl and von Cramon (2001), coherence and cohesion interacted, such that the addition of a connective signaling cohesion had a bigger effect in the less-coherent passages than in the coherent passages. Contrasts of the four inference conditions with fixation showed decreased activation in $\mathrm{RH}$ homologues of LH language areas (inferior frontal gyrus and anterior and posterior regions of the middle temporal gyrus) when connectives were present in the coherent passages and an increase in activation in $\mathrm{RH}$ homologues when connectives were present in the less-coherent passages. Patterns of $\mathrm{RH}$ activation in the four inference conditions versus fixation are illustrated in Fig. 2.

The direct test of this interaction, the subtraction of the cohesion effect (connective-no connective) in the coherent passages (moderately related) from the cohesion effect in the less-coherent (distantly related) passages, yielded reliably greater activation in the right angular gyrus extending into superior parietal regions, in the right middle temporal gyrus, and in the right caudate nucleus, extending into the anterior cingulum. Peak MNI coordinates, Brodmann's areas, and $T$-values for regions of significant activation in each of the conditions versus fixation, for the main effects of coherence and cohesion, and for the interaction are listed in Table 3.

\subsection{Coherence and cohesion in the left hemisphere}

Distribution of activation analyses at the group level showed relatively consistent activation of LH language regions across the four passage types. This overlap of activation is illustrated in Fig. 3, where regions shown in blue depict regions that were active in all of the passage types.

\footnotetext{
2 There was one significant cluster of activation in the right medial temporal lobe in the less-coherent passages without connectives that is difficult to see on the rendered brain because it was a rather small and medial cluster. See Table 3 for coordinates.
}

The interaction between coherence and cohesion was also manifest in changes in LH activation. Specifically, a greater effect of cohesion (connective-no connective) in the less-coherent passages than in the coherent passages was observed in the left angular gyrus, extending into the superior parietal region, and in the left inferior and middle temporal gyri. Peak MNI coordinates, Brodmann's areas, and $T$-values for regions of significant activation in each of the conditions versus fixation, and for the interaction are listed in Table 3.

\subsection{Individual differences in neural efficiency}

Correlations between individual vocabulary size and activation in the context sentences revealed negative correlations between vocabulary size and activation in the right inferior frontal gyrus, as well as in left temporal and left inferior occipital regions (including the fusiform gyrus) and bilateral cerebellum. There were no regions in which positive correlations between vocabulary size and activation were observed for the context sentences.

This increased efficiency (decreased activation) with increased language skill also resulted in negative correlations, primarily in the $\mathrm{RH}$, during the inference window of each of the four passage types. The correlation between $\mathrm{RH}$ activation and vocabulary size was most obvious in the two most difficult passage types, when coherent passages did not have connectives and when less-coherent passages did have connectives. Reliable negative correlations between vocabulary size and activation in the RH homologue of Broca's area (IFG) were observed in both passage types. In addition, when comprehending coherent passages without connectives (one of the difficult passage types), less-skilled readers showed greater activation in the RH homologue of Wernicke's area (PSTG) and in the RH middle frontal and orbital frontal gyri. Negative correlations with vocabulary size and activation were also observed on the border of the right inferior occipital region and the cerebellum (slightly inferior to the word form area) during comprehension of all passage types; however the cluster did not reach the significant 12 voxel threshold during comprehension of the moderately related passages without clause connectives. To illustrate individual differences in neural efficiency in the $\mathrm{RH}$, reliable negative correlations between reading skill and activation during context sentences (blue), coherent/incohesive inferences (red), incoherent/ 
Table 3

Clusters of activation from whole-brain analysis for each of the passage conditions versus fixation and for the interaction between coherence and cohesion.

\begin{tabular}{|c|c|c|c|c|c|c|}
\hline \multirow[t]{2}{*}{ Cortical region } & \multirow[t]{2}{*}{ Brodmann's area (Peak) } & \multirow[t]{2}{*}{ Cluster size } & \multirow[t]{2}{*}{ Peak $T$-value } & \multicolumn{3}{|c|}{ MNI coordinates } \\
\hline & & & & $x$ & $y$ & $z$ \\
\hline \multicolumn{7}{|l|}{ (A) Coherent/cohesive-fixation } \\
\hline Left temporal/parietal & 21 & 1829 & 6.30 & -48 & -34 & -8 \\
\hline Left middle/inferior temporal & 21 & 90 & 4.85 & -58 & -2 & -26 \\
\hline Left inferior frontal & 47 & 1492 & 7.23 & -50 & 36 & -14 \\
\hline Left middle frontal/precentral & 6 & 282 & 4.76 & -38 & 4 & 60 \\
\hline Right mid temporal/hippocampus & 21 & 588 & 5.05 & 50 & -30 & -6 \\
\hline Right anterior middle temporal & 21 & 13 & 4.29 & 58 & 6 & -24 \\
\hline Right inferior frontal & 45 & 21 & 4.51 & 62 & 24 & 16 \\
\hline Left SMA & 6 & 108 & 4.42 & -8 & 14 & 62 \\
\hline Left hippocampus & 27 & 43 & 4.08 & -26 & -34 & -2 \\
\hline Left basal ganglia & & 13 & 4.04 & -6 & 10 & 16 \\
\hline Left inferior temporal/fusiform & 37 & 14 & 3.98 & -42 & -42 & -26 \\
\hline Bilateral occipital & 18 & 6304 & 9.41 & 4 & -100 & -2 \\
\hline \multicolumn{7}{|l|}{ (B) Coherent/incohesive-fixation } \\
\hline \multicolumn{7}{|l|}{ Left middle temporal/inferior } \\
\hline Frontal/bilateral occipital & 17 & 16,915 & 11.16 & 6 & -100 & -2 \\
\hline Left medial/superior frontal/SMA & 6 & 692 & 6.22 & -4 & 12 & 56 \\
\hline Left medial/superior frontal & 10 & 157 & 5.01 & -12 & 62 & 32 \\
\hline Right middle/superior temporal & 21 & 925 & 6.33 & 52 & -30 & -4 \\
\hline Right inferior frontal & 45 & 29 & 4.53 & 62 & 22 & 8 \\
\hline Right inferior frontal & 9 & 20 & 4.49 & 38 & 24 & 26 \\
\hline Right inferior/orbital frontal & 47 & 13 & 4.41 & 48 & 30 & -16 \\
\hline Right anterior middle/superior temporal & 21 & 149 & 6.54 & 58 & 6 & -22 \\
\hline Left precentral & 6 & 14 & 4.31 & -34 & -26 & 68 \\
\hline Left superior frontal & 6 & 34 & 4.22 & -24 & -6 & 72 \\
\hline Left postcentral & 3 & 50 & 4.11 & -40 & -26 & 56 \\
\hline Left occipital/parietal & 7 & 41 & 5.64 & -24 & -58 & 36 \\
\hline Right angular gyrus/superior parietal & 7 & 26 & 4.40 & 34 & -60 & 48 \\
\hline Right basal ganglia & & 169 & 4.39 & 18 & 8 & 6 \\
\hline Right occipital/cerebellum & 19 & 157 & 4.38 & 12 & -56 & -4 \\
\hline Left cerebellum & & 34 & 4.28 & 4 & -44 & -22 \\
\hline Right cerebellum & & 12 & 3.93 & 30 & -58 & -26 \\
\hline Left hippocampus/parahippocampus & 27 & 790 & 5.00 & -24 & -34 & -4 \\
\hline Right hippocampus & 27 & 114 & 4.85 & 30 & -34 & 0 \\
\hline \multicolumn{7}{|l|}{ (C) Less-coherent/cohesive-fixation } \\
\hline Left middle temporal & 21 & 1707 & 6.06 & -60 & -34 & -4 \\
\hline Left inferior frontal & 46 & 1965 & 7.85 & -50 & 20 & 24 \\
\hline Left medial/superior frontal & 8 & 76 & 4.64 & -10 & 42 & 56 \\
\hline Right middle temporal/hippocampus & & 816 & 6.99 & 32 & -34 & 2 \\
\hline Right anterior temporal & 21 & 18 & 5.08 & 56 & 8 & -24 \\
\hline Right inferior frontal & 45 & 19 & 5.03 & 62 & 24 & 14 \\
\hline Right angular gyrus & 7 & 15 & 4.07 & 30 & -64 & 42 \\
\hline Left inferior temporal/fusiform & 37 & 81 & 4.36 & -42 & -50 & -18 \\
\hline Left SMA & 6 & 121 & 4.41 & -8 & 14 & 60 \\
\hline Left hippocampus & 34 & 12 & 4.09 & -22 & -10 & -14 \\
\hline Left hippocampus & 27 & 227 & 5.58 & -26 & -32 & -4 \\
\hline Left basal ganglia & & 48 & 5.10 & -8 & 14 & 14 \\
\hline Bilateral occipital & 17 & 6192 & 10.13 & 6 & -100 & -2 \\
\hline (D) Less-coherent/incohesive-fixation & & & & & & \\
\hline Left middle temporal & 21 & 19 & 4.84 & -60 & -2 & -24 \\
\hline Left middle temporal & 21 & 245 & 4.45 & -48 & -32 & -6 \\
\hline Left middle temporal & 22 & 45 & 4.09 & -52 & -48 & 12 \\
\hline Left inferior frontal & 45 & 349 & 5.71 & -54 & 22 & 22 \\
\hline Right middle temporal & 21 & 65 & 4.11 & 50 & -30 & -6 \\
\hline Bilateral occipital & 18 & 3834 & 8.41 & -22 & -90 & -16 \\
\hline Right cerebellum & 19 & 23 & 4.14 & 36 & -78 & -22 \\
\hline (E) All coherent-all less-coherent & & & & & & \\
\hline Left anterior temporal & 22 & 139 & 5.72 & -58 & 2 & 2 \\
\hline Left middle/superior frontal & 10 & 135 & 5.18 & -20 & 46 & 16 \\
\hline Left middle temporal & 21 & 27 & 4.3 & -66 & -26 & -4 \\
\hline Left posterior temporal/parietal & 40 & 14 & 4.25 & -48 & -32 & 24 \\
\hline Right superior temporal & 22 & 20 & 3.98 & 58 & -10 & 0 \\
\hline Left superior medial frontal & 8 & 15 & 3.9 & -16 & 36 & 54 \\
\hline Left caudate and insula & & 118 & 4.65 & -14 & 22 & -2 \\
\hline Left middle temporal & 21 & 12 & 3.9 & -54 & 4 & -18 \\
\hline Left cingulum & 24 & 14 & 4.46 & -10 & -6 & 38 \\
\hline Left precuneus & 31 & 93 & 4.48 & -20 & -50 & 6 \\
\hline Left fusiform/occipital & 37 & 219 & 6.58 & -22 & -48 & -10 \\
\hline Left middle occipital & 19 & 89 & 5.35 & -36 & -80 & 16 \\
\hline Left occipital & 18 & 260 & 4.52 & -18 & -84 & 18 \\
\hline Right fusiform & 19 & 60 & 4.46 & 28 & -68 & -6 \\
\hline
\end{tabular}




\begin{tabular}{|c|c|c|c|c|c|c|}
\hline \multirow[t]{2}{*}{ Cortical region } & \multirow[t]{2}{*}{ Brodmann's area (Peak) } & \multirow[t]{2}{*}{ Cluster size } & \multirow[t]{2}{*}{ Peak $T$-value } & \multicolumn{3}{|c|}{ MNI coordinates } \\
\hline & & & & $x$ & $y$ & $z$ \\
\hline Right posterior temporal/occipital & 19 & 68 & 4.41 & 44 & -82 & 12 \\
\hline Right occipital & 19 & 17 & 4.28 & 12 & -84 & 36 \\
\hline Right fusiform/occipital & 19 & 15 & 3.98 & 20 & -48 & -12 \\
\hline Right occipital & 19 & 14 & 3.98 & 16 & -94 & 26 \\
\hline \multicolumn{7}{|l|}{ (F) All cohesive-all incohesive } \\
\hline Left middle temporal & 21 & 50 & 4.29 & -60 & -34 & -4 \\
\hline Precuneus & 31 & 14 & 4.06 & 30 & -48 & 6 \\
\hline Left occipital & 18 & 345 & 6.09 & -2 & -92 & 2 \\
\hline Right occipital & 17 & 33 & 4.46 & 18 & -76 & 6 \\
\hline Left inferior occipital & 18 & 14 & 4.14 & -28 & -84 & -4 \\
\hline \multicolumn{7}{|l|}{ (G) All incohesive $>$ cohesive } \\
\hline Middle/posterior cingulum & 31 & 256 & 4.94 & -2 & -28 & 36 \\
\hline Medial orbital frontal & 32 & 50 & 4.26 & 2 & 46 & -6 \\
\hline Right parietal & 40 & 24 & 4.26 & 56 & -34 & 50 \\
\hline \multicolumn{7}{|c|}{ (H) Interaction: less-coherent cohesive $>$ incohesive - coherent cohesive $>$ incohesive } \\
\hline Right angular gyrus/parietal & 7 & 230 & 5.54 & 32 & -74 & 54 \\
\hline Right inferior temporal & 37 & 12 & 3.81 & 62 & -48 & -12 \\
\hline Left angular gyrus/parietal & 7 & 459 & 5.57 & -36 & -70 & 52 \\
\hline Left inferior/middle temporal & 37 & 13 & 4.03 & -64 & -48 & -14 \\
\hline Right basal ganglia and cingulum & 13 & 29 & 5.45 & -28 & -30 & 26 \\
\hline
\end{tabular}

\section{Overlap in Left Hemisphere Activation During Inferencing:}

All conditions Coherent/Incohesive Coherent/Cohesive Less-coherent/Cohesive

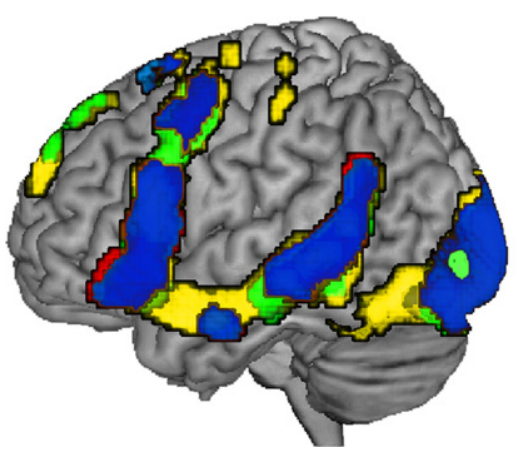

Fig. 3. Activation overlap map showing consistent left hemisphere activation in the four inference conditions (in blue) along with areas of activation for the coherent/ incohesive passages (in yellow), the coherent/cohesive passages (in red), and the incoherent/cohesive passages (in green). Note that there were no areas of unique activation in the incoherent/incohesive passages.

cohesive inferences (yellow) and their overlap is depicted in Fig. 4a. The relation between $\mathrm{RH}$ activation during the coherent passages without connectives and vocabulary size is further depicted in a scatterplot in Fig. 4b, where the average of peak activations across the five clusters in the $\mathrm{RH}$ depicted in $4 \mathrm{a}$ was computed on an individual subject level. MNI coordinates for the peaks of the reliably correlated regions and the corresponding Brodmann's areas are listed in Table 4 . No positive correlations between vocabulary size and RH activation were observed.

\subsection{Neural efficiency and left hemisphere activation}

Correlations between vocabulary size and LH activation were observed during comprehension of less-coherent passages only. When clause connectives were presented, explicitly cuing a relationship between the two sentences, reliable negative correlations between vocabulary size and activation were found in left temporal and parietal regions (including Wernicke's area) and in the left inferior occipital region, extending into the cerebellum. However, when no clause connectives were presented, reliable positive correlations between vocabulary size and activation were found in the left parietal region. Correlations between vocabulary size and LH activation are depicted in Fig. 5. MNI coordinates and Brodmann's areas for the peaks of the reliably correlated regions are presented in Table 4.

\subsection{Individual differences in coherence and cohesion effects}

Moderately related passages elicited more activation across participants than did distantly related passages, resulting in main effects of Coherence in left inferior frontal and left temporal ROIs. This effect was greatest in skilled readers, resulting in reliable Coherence $\times$ Skill interactions in the same ROIs, as well as in the medial frontal ROI. Follow-up analyses found positive correlations between coherence effects (moderately - distantly related passages) and vocabulary size in left inferior-inferior-frontal $[r(18)=.59]$, left middle-inferior-frontal $[r(18)=.51]$, left anterior temporal $[r(18)=.58]$, left middle temporal $[r(18)=.54]$, left posterior superior temporal $[r(18)=.60]$, and medial frontal $[r(18)=.51]$ ROIs. Passages with clause connectives also elicited more activation than passages without connectives, resulting in a main effect of Cohesion in the left anterior temporal ROI. This effect was greatest in less-skilled readers, resulting in a reliable Cohesion $\times$ Skill interaction in the same ROI $[r(18)=-.47]$. Consistent with the whole brain voxel-wise analysis, volumetric analyses revealed an interaction between coherence and cohesion, such that the cohesion effect (additional activation with connectives) was much greater in incoherent passages than in coherent ones. This Coherence $\times$ Cohesion interaction was significant in the left middle-inferior frontal ROI, in the left superior-inferior frontal ROI and in the right middle-inferior frontal ROI. This interaction also varied with individual language skill, such that less-skilled readers had greater cohesion effects in the less-coherent passages than skilled readers, but there was either no differential effect of cohesion in the coherent passages, or the relationship was positive. This reliable Coherence $\times$ Cohesion $\times$ Skill interaction resulted in reliable negative correlations between the Cohesive Effect (connective-no connective) in incoherent passages but not in the coherent passages in the left middle-inferior frontal ROI $[r(18)=-.60$ and .13 respectively], in the left superior-inferior frontal ROI $[r(18)=-.68$ and .14 respectively] and in the right 
A

Increased Right Hemisphere Activation in LessSkilled Readers during Passage Comprehension
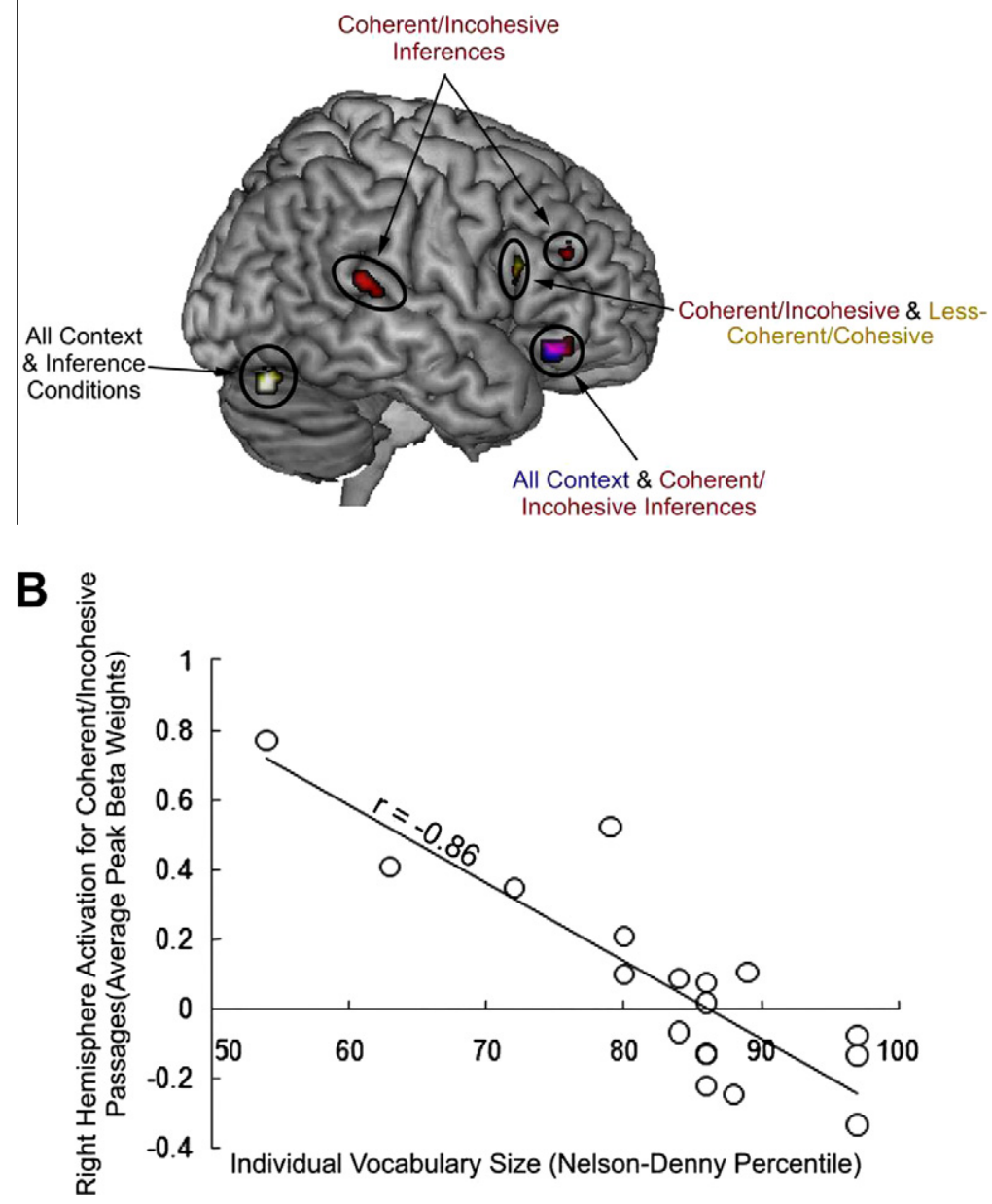

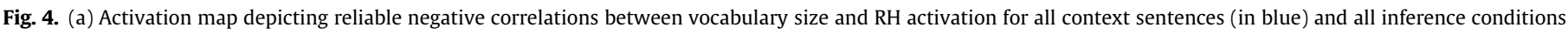

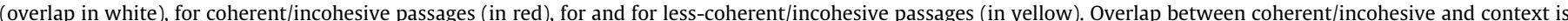

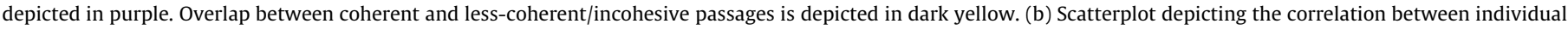
vocabulary size and RH activation during coherent/incohesive inferences.

middle-inferior frontal ROI $[r(18)=-.49$ and .50 respectively]. ANOVA statistics for ROI analyses are listed in Table 5.

\subsection{Individual differences in network synchronization}

Across all passage types, a trend suggesting higher synchronization in skilled readers was observed across most network pairs; however the positive correlations did not reach significance. There were no negative correlations between functional connectivity and vocabulary size observed. Average functional connectivities for all functional networks pairs across all passage types are listed in $\mathrm{Ta}$ ble 6 , along with correlations between these connectivities and vocabulary size.

Functional connectivity results also showed an interaction between reader characteristics and text characteristics, such that Coherence and Cohesion effects interacted with language skill. Consistent with the volumetric analyses, coherence effects (coherent $>$ less-coherent) were biggest in skilled readers (with some less-skilled readers showing less-coherent $>$ coherent) resulting in reliable Coherence $\times$ Skill interactions within the LH temporal/ semantic network and between the $\mathrm{RH}$ frontal/selection and $\mathrm{RH}$ temporal/semantic networks. Also consistent with the volumetric analyses, Cohesion Effects were largest in less-skilled readers, resulting in reliable Cohesion $\times$ Skill interactions within the $\mathrm{LH}$ temporal/semantic network, between the LH and RH temporal/ semantic networks, between the LH temporal/semantic network and the LH anterior temporal/integration network, between the medial frontal/coherence monitoring and LH anterior temporal/ integration networks, and between the medial frontal/coherence monitoring and RH temporal/semantic networks. ANOVA and follow-up statistics for functional connectivity analyses are listed in Table 7.

\section{Discussion}

\subsection{The role of the right hemisphere in inferential process}

The results of this study suggest that the role of the RH in inference generation varies as a function of characteristics that influence the ease with which a reader can comprehend texts. Lessskilled readers, for example, recruited reliably more RH resources across conditions. At the group level, $\mathrm{RH}$ activation varied as a function of text characteristics (coherence and cohesion) that have been shown to influence the difficulty of passage comprehension 
Table 4

Significant correlations with vocabulary size and brain activation during passage comprehension.

\begin{tabular}{|c|c|c|c|c|c|c|}
\hline \multirow[t]{2}{*}{ Cortical region } & \multirow[t]{2}{*}{ Brodmann's area (Peak) } & \multirow[t]{2}{*}{ Cluster size } & \multirow[t]{2}{*}{ Peak $T$-value } & \multicolumn{3}{|c|}{ MNI coordinates } \\
\hline & & & & $x$ & $y$ & $z$ \\
\hline \multicolumn{7}{|c|}{ (A) Negative correlation with reading skill: context sentence only } \\
\hline Right inferior orbital frontal & 11 & 36 & 4.64 & 38 & 34 & -12 \\
\hline Left middle superior temporal & 40 & 17 & 3.98 & -52 & -48 & 20 \\
\hline Left fusiform/inferior occipital/cerebellum & 19 & 93 & 5.29 & -38 & -76 & -22 \\
\hline Left occipital & 37 & 29 & 4.41 & -52 & -72 & -4 \\
\hline Right inferior occipital/cerebellum & 19 & 18 & 4.32 & 38 & -74 & -26 \\
\hline \multicolumn{7}{|c|}{ (B) Negative correlation with reading skill: coherent/cohesive inference condition } \\
\hline Right inferior occipital/cerebellum & 19 & 17 & 4.42 & 38 & -74 & -24 \\
\hline \multicolumn{7}{|c|}{ (C) Negative correlation with reading skill: coherent/incohesive inference condition } \\
\hline Right inferior frontal & 45 & 40 & 5.02 & 42 & 18 & 16 \\
\hline Right middle frontal & 9 & 19 & 4.38 & 32 & 36 & 28 \\
\hline Right inferior orbital frontal & 47 & 35 & 5.05 & 38 & 34 & -10 \\
\hline Right superior posterior temporal & 22 & 17 & 4.14 & 66 & -38 & 14 \\
\hline \multicolumn{7}{|c|}{ (D) Negative correlation with reading skill: less-coherent/cohesive inference condition } \\
\hline Right inferior frontal & 45 & 52 & 4.43 & 42 & 20 & 20 \\
\hline Right parietal & 40 & 17 & 4.42 & 32 & -48 & 48 \\
\hline Right inferior occipital/cerebelum & 19 & 40 & 5.27 & 40 & -74 & -24 \\
\hline Left middle/superior temporal and parietal & 40 & 51 & 5.17 & -50 & -50 & 20 \\
\hline Left parietal & 40 & 12 & 4.11 & -66 & -36 & 30 \\
\hline Left inferior occipital, fusiform, cerebellum & 19 & 48 & 4.84 & -42 & -74 & -20 \\
\hline Left inferior/middle occipital & 19 & 45 & 4.10 & -38 & -70 & 4 \\
\hline \multicolumn{7}{|c|}{ (E) Negative correlation with reading skill: less-coherent/incohesive inference condition } \\
\hline Right inferior occipital/cerebellum & 19 & 12 & 4.54 & 38 & -76 & -24 \\
\hline \multicolumn{7}{|c|}{ (F) Positive correlation with reading skill: less-coherent/incohesive inference condition } \\
\hline Left angular gyrus & 39 & 53 & 4.56 & -52 & -74 & 30 \\
\hline
\end{tabular}

\section{Individual Differences in Left Hemisphere Activation during Passage Comprehension}

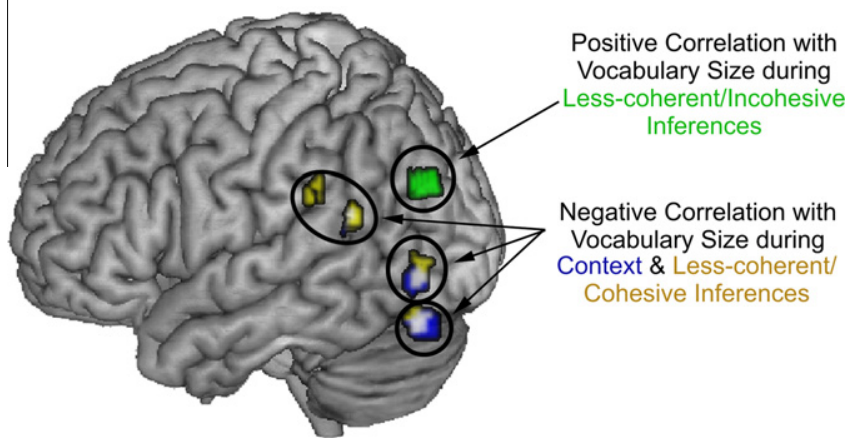

Fig. 5. Activation map depicting reliable negative correlations between vocabulary size and LH activation for all context sentences (in blue) and for less-coherent/ cohesive inferences (in yellow). Overlap between context and less-coherent/ cohesive inferences is in white. Reliable positive correlation between vocabulary size and LH activation during less-coherent/incohesive passages is shown in green.

(Ferstl \& von Cramon, 2001; Keenan et al., 1984; Mason \& Just, 2004; Myers et al., 1987), with highest RH activation observed in the most difficult passages (coherent/incohesive and lesscoherent/cohesive). Our findings, combined with inconsistencies in previous research, highlight the need to systematize observed variability in RH language contributions. We argue that our understanding of $\mathrm{RH}$ contributions to reading comprehension will advance more rapidly if we shift focus from identifying static characterizations of such contributions towards understanding the dynamic factors that relate to conditions under which $\mathrm{RH}$ processes are evoked.

The results described here are consistent with the dynamic $\mathrm{RH}$ spillover hypothesis, and provide additional evidence that the role of the RH in discourse processes is modulated by linguistic skill. These results are also consistent with previous research on individ- ual differences in sentence comprehension (Prat, Keller, et al. 2007; Prat \& Just, in press), lexical ambiguity resolution (Mason \& Just, 2007), and discourse representation (Prat, Long, et al., 2007) suggesting that during language comprehension, less-skilled readers utilize the $\mathrm{RH}$ more so than do skilled readers. It is possible that such individual differences in RH language involvement contribute to the inconsistencies observed in the literature on RH linguistic function. The dynamic spillover hypothesis of RH language function suggests a novel research approach, focusing not on identifying distinctively $\mathrm{RH}$ linguistic specializations, but on examining the conditions under which RH function is evoked.

According to Jung-Beeman's coarse coding theory, the RH's coarsely-coded semantic representations are drawn upon during inference generation to activate, select, and integrate distant semantic relations (e.g., Jung-Beeman, 2005). Our group-level results are somewhat consistent with a coarse coding theory, with right middle temporal lobe activation in all inference conditions, and right inferior frontal and anterior temporal activation in all passages except for the condition where readers were least likely to successfully select and integrate an inference, in the lesscoherent/incohesive passages. We find increased activation in less-skilled readers, however, in all of the semantic processing areas described by Jung-Beeman and colleagues. To integrate these findings, one either needs to understand why a less-skilled reader would rely more heavily on coarse-coding processes or to elaborate the hypothesis with a consideration of RH language contributions varying as a function of processing demands experienced by the individual. An alternate approach to integrating the findings is to assume that coarse coding is an effect of the spillover process. In other words, the RH's representation of semantic information could be coarser than the LH's because it has less experience with language (due to less consistent recruitment).

\subsection{The Neural Basis of Reading Comprehension Abilities}

One goal of this experiment was to explore potential biological constraints to inferential processes during discourse comprehension. 
Table 5

ANOVA statistics for volumetric analyses of regions of interest.

\begin{tabular}{|c|c|c|c|}
\hline Region of interest & df & $F$ & MSE \\
\hline \multicolumn{4}{|c|}{ Main effect of coherence (coherent > less-coherent) } \\
\hline Left inferior frontal (inf) & 1,16 & 7.36 & 16,644 \\
\hline Left inferior frontal (mid) & 1,16 & 5.67 & 35,420 \\
\hline Left anterior temporal & 1,16 & 6.54 & 9390 \\
\hline Left middle temporal & 1,16 & 5.20 & 123,277 \\
\hline Left posterior superior temporal & 1,16 & 8.00 & 167,954 \\
\hline \multicolumn{4}{|l|}{ Coherence $\times$ Skill interaction } \\
\hline Left inferior frontal (inf) & 1,16 & 8.67 & 16,644 \\
\hline Left inferior frontal (mid) & 1,16 & 5.72 & 35,420 \\
\hline Left anterior temporal & 1,16 & 7.93 & 9390 \\
\hline Left middle temporal & 1,16 & 5.20 & 123,277 \\
\hline Left posterior superior temporal & 1,16 & 8.93 & 167,954 \\
\hline Medial frontal & 1,16 & 5.57 & 41,396 \\
\hline \multicolumn{4}{|c|}{ Main effect of cohesion (cohesive > incohesive) } \\
\hline Left anterior temporal & 1,16 & 6.03 & 9390 \\
\hline \multicolumn{4}{|l|}{ Cohesion $\times$ Skill interaction } \\
\hline Left anterior temporal & 1,16 & 4.50 & 9390 \\
\hline \multicolumn{4}{|l|}{ Coherence $\times$ Cohesion interaction } \\
\hline Left inferior frontal (mid) & 1,16 & 6.23 & 25,597 \\
\hline Left inferior frontal (sup) & 1,16 & 6.87 & 10,288 \\
\hline Right inferior frontal (mid) & 1,16 & 10.07 & 20,911 \\
\hline \multicolumn{4}{|c|}{ Coherence $\times$ Cohesion $\times$ Skill Interaction } \\
\hline Left inferior frontal (mid) & 1,16 & 5.88 & 25,597 \\
\hline Left inferior frontal (sup) & 1,16 & 5.59 & 10,288 \\
\hline Right inferior frontal (mid) & 1,16 & 9.74 & 20,911 \\
\hline
\end{tabular}

Previous research suggests that reading skill is related to the likelihood that an individual will generate optional, elaborative inferences when comprehending texts (Long et al., 1994, 1997). The results of this study showed that less-skilled readers had less-efficient neural processes when reading. Specifically, lessskilled readers had reliably higher activation in brain regions including right hemisphere homologues of left hemisphere language regions and left posterior temporal/parietal and regions, than skilled readers, even when reading the relatively undemanding context sentences. This is consistent with our prediction that less-skilled readers may fail to generate optional inferences because of limitations in available neural resources. In a recent neuroimaging investigation of constraints on sentence processing, we showed that ability to comprehend complex sentences was related to the ability to recruit additional resources in the face of increasing task demands (Prat \& Just, in press). Differences in readers' engagement in elaborative inference processing, then, may arise because of a lack of available resources for inferential computations.

The results from this study did not show significantly higher functional connectivity in skilled readers, but non-significant positive correlations were observed between most network pairs (as shown in Table 6). Previous research looking at individual differences in functional connectivity in extreme groups of high- and low-capacity readers showed that high-capacity readers had better synchronization across sentence conditions than did low-capacity readers (Prat, Keller, et al., 2007). A recent study investigating both working memory capacity and reading experience as predictors of functional connectivity found that only working memory capacity was related to individual differences in connectivity, such that higher-capacity individuals had better modulation of activation with changing task demands. Our results showing higher increase in connectivity in coherent (moderately related) versus lesscoherent (distantly related) passages in skilled readers is somewhat consistent with this view, however there are cognitive factors (e.g., whether inferences were drawn in less-coherent passages) that may also explain these results.

Our results also showed important individual differences in modulation of the left hemisphere language regions as a function of coherence and cohesion. Skilled readers showed greater coherence

Table 6

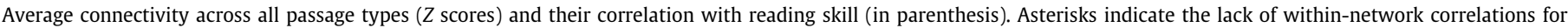
functional units that only contained one ROI.

\begin{tabular}{|c|c|c|c|c|c|c|c|}
\hline & 1 & 2 & 3 & 4 & 5 & 6 & 7 \\
\hline 1. LH frontal/selection & $1.0(.20)$ & & & & & & \\
\hline 2. LH temporal/semantic & $.92(.05)$ & $1.07(.19)$ & & & & & \\
\hline 3. LH ant temporal/integration & $89(.18)$ & $.88(.18)$ & $*$ & & & & \\
\hline 4. $\mathrm{RH}$ frontal/selection & $.87(.46)$ & $.73(.33)$ & $.74(.23)$ & $.83(.25)$ & & & \\
\hline 5. RH temporal/semantic & $.75(.29)$ & $.91(.27)$ & $.80(.20)$ & $.79(.21)$ & .94 & & \\
\hline 6. Medial frontal/coherence & $.71(.28)$ & $.76(.30)$ & $.68(.20)$ & $.66(.35)$ & $.60(.31)$ & $*$ & \\
\hline 7. Precuneus/memory & $.51(-.09)$ & $.66(-.13)$ & $.54(.07)$ & $.55(.05)$ & $.66(-.01)$ & $.56(.42)$ & * \\
\hline
\end{tabular}

Table 7

ANOVA statistics for network connectivity analyses.

\begin{tabular}{|c|c|c|c|c|}
\hline Network(s) & $\underline{\mathrm{df}}$ & $F$ & MSE & Follow-up analyses \\
\hline \multicolumn{5}{|l|}{ Coherence } \\
\hline Within left temporal & 1,15 & 9.51 & .010 & \\
\hline Right frontal: right temporal & 1,13 & 8.58 & .014 & \\
\hline Coherence $\times$ Skill & & & & Correlation: skill and coherence effect \\
\hline Within left temporal & 1,15 & 7.95 & .010 & $r(17)=.59$ \\
\hline Right frontal: right temporal & 1,13 & 8.52 & .014 & $r(15)=.63$ \\
\hline \multicolumn{5}{|l|}{ Main effect of cohesion } \\
\hline Left temporal: right temporal & 1,16 & 6.35 & .020 & \\
\hline Left ant temporal: left post temporal & 1,15 & 6.40 & .013 & \\
\hline Monitor: left ant temporal & 1,13 & 7.46 & .018 & \\
\hline Monitor: right temporal & 1,13 & 4.70 & .070 & \\
\hline Cohesion $\times$ Skill & & & & Correlation: skill and cohesion effect \\
\hline Within left temporal & 1,15 & 5.17 & .023 & $r(17)=-.51$ \\
\hline Left temporal: right temporal & 1,16 & 6.78 & .020 & $r(18)=-.55$ \\
\hline Left ant temporal: left post temporal & 1,15 & 7.45 & .013 & $r(17)=-.58$ \\
\hline Monitor: left ant temporal & 1,13 & 7.68 & .018 & $r(15)=-.61$ \\
\hline Monitor: right temporal & 1,13 & 4.70 & .070 & $r(15)=-.52$ \\
\hline
\end{tabular}


effects (increased activation for coherent over less-coherent passages) in left hemisphere language regions and in medial frontal coherence monitoring regions than did less-skilled readers. In contrast, less-skilled readers showed greater cohesion effects (increased activation for passages with connectives over passages without connectives) than did skilled readers in the left anterior temporal regions.

These results seem to be consistent with behavioral research showing that individual differences in reading skill at the college level are manifest by differences in optional, elaborative and integrative processing of texts. Increased sensitivity to coherence throughout the left dominant language network (irrespective of explicit information cuing causal relatedness) coupled with increased modulation of the medial frontal coherence monitoring regions, suggests that skilled readers are more sensitive to causal relatedness manipulations between sentences than are less-skilled readers. In the less-coherent/incohesive passages, skilled readers activated more left parietal lobe than did skilled readers, possibly suggesting that they search for coherence in these trials and try to generate associations/possible scenarios relating the two sentences, whereas less-skilled readers just process the sentences as independent statements. In addition, less-skilled readers had bigger activation changes in the left temporal ROI, and a greater increase in synchronization between several areas including the medial frontal/coherence monitoring node and bilateral tempo$\mathrm{ral} /$ semantic networks when connectives were present, suggesting that the explicit cuing of causal relations had a larger influence on their processing of texts than it did on skilled readers.

Two previous neuroimaging experiments have examined the neural basis of individual differences in inference generation as a function of individual working memory capacity (Virtue et al., 2006, 2008). In their first report, Virtue et al. (2006) found that skilled readers had larger "inference effects," indexed by the difference between brain activation resulting from listening to stories that did not require inferences compared to stories that did require inferences. The authors did not report any relation between $\mathrm{RH}$ activation and working memory capacity, and they did not correlate skill with the total amount of activation in any reading condition versus baseline. It is possible that low-capacity individuals had greater RH activation in both explicit and inference conditions. In a second experiment, however, Virtue et al. (2008) did find increased inference effects in $\mathrm{RH}$ homologues of high-capacity readers. We offer the following suggestions as to why these results may be inconsistent with our own. First, both of Virtue's experiments involved auditory presentation of the stories, which is known to elicit more bilateral activation, especially in the temporal lobes, than does visual presentation (Buchweitz, Mason, Tomitch, \& Just, 2009; Carpentier et al., 2001; Constable et al., 2004; Jobard, Vigneau, Mazoyer, \& Tzourio-Mazoyer, 2007; Michael, Keller, Carpenter, \& Just, 2001). Unlike our reading experiments, the auditory presentation style also controls for the rate at which individuals receive linguistic input. It is possible that our efficiency results occur, in part, because of reading time differences between skilled and less-skilled readers; however while skilled readers read faster on average than less-skilled readers, during critical points of passages (e.g., ambiguous sections, or when inferences need to be drawn), skilled readers are more likely to slow down then are lessskilled readers (e.g., Long \& Prat, 2008). To explore this possibility, we correlated one index of reading rate (response time to filler passages) with vocabulary size and found a non-significant trend toward slower reading times in skilled readers $[r(18)=-.34$, $p=.17]$. We also used these response times as a regressor to predict activation during context sentences and found that longer response times were associated with small clusters of activation primarily in motor and orbital frontal regions that did not overlap with our efficiency results. Another difference between the existing literature and the current experiment is that Virtue's experiments involve inference-specific activation (contrasts with explicit passages) whereas ours involve contrasts with fixation; therefore, if, as described above, efficiency differences occur in explicit passages in Virtue's experiments, they are subtracted out. In addition, Virtue's analyses involve split-group comparisons of high- and low-capacity individuals whereas ours involve correlations. Finally, Virtue's analyses investigate individual differences in working memory capacity, which are correlated with, but separable from, individual differences in reading skill, both in their behavioral implications and in their neural underpinnings (Prat \& Just, in press). One goal for future research is to examine individual differences in discourse comprehension processes as a function of both vocabulary size and working memory capacity to disentangle some of the issues raised here.

The current experiment explores the nature of capacity constraints during inferential processes by examining patterns of activation in individuals as a function of vocabulary size. We found that skilled reading was underpinned by more-efficient neural activation (indexed primarily by less recruitment of $\mathrm{RH}$ homologues). These results extend our previous findings suggesting that network-level characterizations of functions enrich our understanding of the neural underpinnings of language comprehension processes.

\section{Summary}

According to the dynamic spillover hypothesis, the RH is more apt to be activated in certain situations (say, in the comprehension of inferences) not because a particular type of process is called for, but because the situation calls for a greater amount of processing than the LH alone can produce. The spillover hypothesis proposes that many of the language functions in the $\mathrm{RH}$ are less efficient counterparts of LH functions, which are evoked first and therefore more frequently. The coarse coding attributed to $\mathrm{RH}$, as described by Jung-Beeman and colleagues (Beeman, 1993b; Beeman, Bowden, \& Gernbacher, 2000; Jung-Beeman, 2005), may be a manifestation of the lower efficiency and lower frequency of use of the RH. In other words, coarse coding may arise because the $\mathrm{RH}$ has less regular experience with language than does the $\mathrm{LH}$. This is not to say that the RH does not have distinctive and valuable processing characteristics that in some circumstances could be more effective than their LH counterparts, nor does our proposal deny the possibility that there are computational functions for which RH is more efficient. Rather, we argue that the understanding of the role of $\mathrm{RH}$ in language may benefit from the perspective that its activation is a manifestation of resource-driven dynamic allocation of function. This dynamic perspective make sense of the inconsistencies in previous characterizations of $\mathrm{RH}$ language function at the group level and provides a framework for investigating the conditions under which the $\mathrm{RH}$ contributes to inferential processes and more generally to language comprehension at all levels.

\section{Acknowledgments}

This research was supported by the National Institute of Mental Health Grant number MH029617 and by a National Institute on Deafness and Communication Disorders Pathway to Independence award (DC009634). We would like to thank Jennifer Moore and Andrea Stocco for their helpful comments on various versions of this manuscript.

\section{References}

Beeman, M. (1993a). Coherence inferencing and structure building in the cerebral hemispheres. Brain and Language, 44(4), 470-471. 
Beeman, M. (1993b). Semantic processing in the right-hemisphere may contribute to drawing inferences from discourse. Brain and Language, 44(1), 80-120.

Beeman, M. J., Bowden, E. M., \& Gernbacher, M. A. (2000). Right and left hemisphere cooperation for drawing predictive and coherence inferences during normal story comprehension. Brain and Language, 71(2), 310-336.

Bèuchel, C., Coull, J. T., \& Friston, K. J. (1999). The predictive value of changes in effective connectivity for human learning. Science, 283(5407), 1538-1541.

Brownell, H. H., Potter, H. H., Bihrle, A. M., \& Gardner, H. (1986). Inference deficits in right brain-damaged patients. Brain and Language, 27(2), 310-321.

Buchweitz, A., Mason, R. A., Tomitch, L. M. B., \& Just, M. A. (2009). Brain activation for reading and listening comprehension: An fMRI study of modality effects and individual differences in language comprehension. Psychology \& Neuroscience, 2(2), 111-123.

Carpentier, A., Pugh, K. R., Westerveld, M., Studholme, C., Skrinjar, O., Thompson, J L., et al. (2001). Functional MRI of language processing: Dependence on input modality and temporal lobe epilepsy. Epilepsia, 42(10), 1241-1254.

Chow, H. M., Kaup, B., Raabe, M., \& Greenlee, M. W. (2008). Evidence of frontotemporal interactions for strategic inference processes during language comprehension. NeuroImage, 40, 940-954.

Constable, R. T., Pugh, K. R., Berroya, E., Mencl, W. E., Westerveld, M., Ni, W., et al. (2004). Sentence complexity and input modality effects in sentence comprehension: An fMRI study. Neuroimage, 22(1), 11-21.

Daneman, M., \& Carpenter, P. A. (1980). Individual differences in working memory and reading. Journal of Verbal Learning E Verbal Behavior, 19(4), 450-466.

Daneman, M., \& Merikle, P. M. (1996). Working memory and language comprehension: A meta-analysis. Psychonomic Bulletin Er Review, 3(4), 422-433.

Ferstl, E. C., Neumann, J., Bogler, C., \& von Cramon, D. Y. (2008). The extended language network: A meta-analysis of neuroimaging studies on text comprehension. Human Brain Mapping, 29(5), 581-593.

Ferstl, E. C., \& von Cramon, D. Y. (2001). The role of coherence and cohesion in text comprehension: An event-related fMRI study. Cognitive Brain Research, 11, 325-340.

Friese, U., Rutschmann, R., \& Raabe, M. (2008). Neural indicators of inference processes in text comprehension: An event-related functional magnetic resonance imaging study. Journal of Cognitive Neuroscience, 20(11), 2110-2124.

Friston, K. J. (1994). Functional and effective connectivity in neuroimaging: A synthesis. Human Brain Mapping, 2(1-2), 56-78.

Friston, K. J., Frith, C. D., Frackowiak, R. S., \& Turner, R. (1995). Characterizing dynamic brain responses with fMRI: A multivariate approach. Neuroimage, 2(2) 166-172.

Haier, R. J., Siegel, B. V., Nuechterlein, K. H., Hazlett, E., Wu, J. C., Paek, J., et al. (1988). Cortical glucose metabolic-rate correlates of abstract reasoning and attention studied with positron emission tomography. Intelligence, 12(2), 199-217.

Jobard, G., Vigneau, M., Mazoyer, B., \& Tzourio-Mazoyer, N. (2007). Impact of modality and linguistic complexity during reading and listening tasks. Neuroimage, 34(2), 784-800.

Jung-Beeman, M. (2005). Bilateral brain processes for comprehending natural language. Trends in Cognitive Sciences, 9(11), 712-718.

Just, M. A., \& Carpenter, P. A. (1992). A capacity theory of comprehension: Individual differences in working memory. Psychological Review, 99(1), 122-149.

Just, M. A., Carpenter, P. A., Keller, T. A., Eddy, W. F., \& Thulborn, K. R. (1996). Brain activation modulated by sentence comprehension. Science, 274(5284), 114-116.

Just, M. A., \& Varma, S. (2007). The organization of thinking: what functional brain imaging reveals about the neuroarchitecture of complex cognition. Cogn Affect Behav Neurosci, 7(3), 153-191.

Keenan, J. M., Baillet, S. D., \& Brown, P. (1984). The effects of causal cohesion on comprehension and memory. Journal of Verbal Learning $\mathcal{E}$ Verbal Behavior 23(115-126).

Kuperberg, G. R., Lakshmanan, B. M., Caplan, D. N., \& Holcomb, P. J. (2006). Making sense of discourse: An fMRI study of causal inferencing across sentences. Neuroimage, 33(1), 343-361.

Long, D. L., Oppy, B. J., \& Seely, M. R. (1994). Individual differences in the time course of inferential processing. Journal of Experimental Psychology: Learning, Memory and Cognition, 20(6), 1456-1470.

Long, D. L., Oppy, B. J., \& Seely, M. R. (1997). Individual differences in readers' sentence- and text-level representations. Journal of Memory and Language, 36(1), 129-145.
Long, D. L., \& Prat, C. S. (2008). Individual differences in syntactic ambiguity resolution: Readers vary in their use of plausibility information. Memory $\mathcal{E}$ Cognition, 36(2), 375-391.

Mason, R. A., \& Just, M. A. (2004). How the brain processes causal inferences in text - A theoretical account of generation and integration component processes utilizing both cerebral hemispheres. Psychological Science, 15(1), 1-7.

Mason, R. A., \& Just, M. A. (2006). Neuroimaging contributions to the understanding of discourse processes. In M. Traxler \& M. A. Gernbacher (Eds.), Handbook of psycholinguistics (pp. 765-799). Amsterdam: Elsevier.

Mason, R. A., \& Just, M. A. (2007). Lexical ambiguity in sentence comprehension. Brain Research, 1146, 115-127.

Maxwell, A. E., Fenwick, P. B., Fenton, G. W., \& Dollimore, J. (1974). Reading ability and brain function: A simple-statistical model. Psychological Medicine, 4, 274-280.

McDonald, S., \& Wales, R. (1986). An investigation of the ability to process inferences in language following right hemisphere brain damage. Brain and Language, 29(1), 68-80.

Michael, E. B., Keller, T. A., Carpenter, P. A., \& Just, M. A. (2001). FMRI investigation of sentence comprehension by eye and by ear: Modality fingerprints on cognitive processes. Human Brain Mapping, 13(4), 239-252.

Millis, K. K., \& Just, M. A. (1994). The influence of connectives on sentence comprehension. Journal of Memory and Language, 33, 128-147.

Mitchell, R. L., \& Ross, E. D. (2008). FMRI evidence for the effect of verbal complexity on lateralisation of the neural response associated with decoding prosodic emotion. Neuropsychologia, 46(12), 2880-2887.

Myers, J. L., Shinjo, M., \& Duffy, S. A. (1987). Degree of causal relatedness and memory. Journal of Memory and Language, 26(4), 453-465.

Neubauer, A. J., \& Fink, A. (2009). Intelligence and neural efficiency. Neuroscience and Biobehavioral Reviews, 33, 1004-1023.

Prat, C. S., \& Just, M. A. (in press). Exploring the neural dynamics underpinning individual differences in sentence comprehension. Cerebral Cortex.

Prat, C. S., Keller, T. A., \& Just, M. A. (2007). Individual differences in sentence comprehension: A functional magnetic resonance imaging investigation of syntactic and lexical processing demands. Journal of Cognitive Neuroscience, 19(12), 1950-1963.

Prat, C. S., Long, D. L., \& Baynes, K. (2007). The representation of discourse in the two hemispheres: An individual differences investigation. Brain and Language, 100(3), 283-294.

Reichle, E. D., Carpenter, P. A., \& Just, M. A. (2000). The neural basis of strategy and skill in sentence picture verification. Cognitive Psycology, 40, 261-295.

Reichle, E. D., \& Mason, R. A. (2007). The neural signatures of causal inferences: A preliminary computational account of brain-imaging and behavioral data. In $\mathrm{F}$. Schmalhofer \& C. A. Perfetti (Eds.), Higher level language processes in the brain: Inference and comprehension processes. Mahwah, NJ, US: Lawrence Erlbaum Associates Publishers.

Sieborger, F. T., Ferstl, E. C., \& von Cramon, D. Y. (2007). Making sense of nonsense: An fMRI study of task induced inference processes during discourse comprehension. Brain Research, 77, 91.

St. George, M., Mannes, S., \& Hoffman, J. E. (1997). Individual differences in inference generation: An ERP analysis. Journal of Cognitive Neuroscience, 9(6), 776-787.

Tompkins, C. A. (1991). Redundancy enhances emotional inferencing by right- and left-hemisphere-damaged adults. Journal of Speech and Hearing Research, 34(5), 1142-1149.

Tompkins, C. A., Fassbinder, W., Lehman Blake, M., Baumgaertner, A., \& Jayaram, N. (2004). Inference generation during text comprehension by adults with right hemisphere brain damage: Activation failure versus multiple activation. Journal of Speech, Language, and Hearing Research, 47(6), 1380-1395.

Virtue, S., Haberman, J., Clancy, Z., Parrish, T., \& Jung Beeman, M. (2006). Neural activity of inferences during story comprehension. Brain Research, 1084(1), 104-114.

Virtue, S., Parrish, T., \& Jung-Beeman, M. (2008). Inferences during story comprehension: Cortical recruitment affected by predictability of events and working memory capacity. Journal of Cognitive Neuroscience, 20(12), 2274-2284. 